\title{
Correction to: Modeling the inconsistency in intertemporal choice: the generalized Weibull discount function and its extension
}

\author{
Salvador Cruz Rambaud ${ }^{1}\left(\right.$ I • Isabel González Fernández ${ }^{1}$ • \\ Viviana Ventre $^{2}$ (D)
}

\section{Correction to: Ann Finance https://doi.org/10.1007/s10436-018-0318-3}

In the original publication, the second author's name was incorrect. The correct name should be Isabel González Fernández.

The original article has been corrected.

The original article can be found online at https://doi.org/10.1007/s10436-018-0318-3.

Salvador Cruz Rambaud scruz@ual.es

Isabel González Fernández

igf389@inlumine.ual.es

Viviana Ventre

viviana.ventre@unicampania.it

1 Departamento de Economía y Empresa, Universidad de Almería, La Cañada de San Urbano, s/n, 04120 Almería, Spain

2 Dipartimento di Matematica e Fisica, Università della Campania “Luigi Vanvitelli”, Viale Lincoln, 5, 81100 Caserta, Italy 